\section{Avaliação e incorporação de tecnologias em saúde: processo e metodologia adotados por um hospital universitário de alta complexidade assistencial}

\author{
Evaluation and incorporation of health \\ technologies: process and methodology adopted \\ by a high-complexity care university hospital
}

\author{
Evaluación e incorporación de tecnologías de \\ salud: proceso y metodología adoptados en un \\ hospital universitario de alta complejidad
}

\author{
1 Faculdade de Medicina de \\ Ribeirão Preto, Universidade \\ de São Paulo, Ribeirão Preto, \\ Brasil. \\ Correspondência \\ A. A. Nunes \\ Faculdade de Medicina de \\ Ribeirão Preto, Universidade \\ de São Paulo. \\ Av. Bandeirantes 3900 \\ Ribeirão Preto, SP 14049-900 \\ Brasil. \\ altacilio@fmrp.usp.br
}

\begin{abstract}
The demographic and epidemiological transitions tend to increase the role of hospitals in medical care. Within such organizations, effective, safe, and cost-effective health technologies ensure better quality of care and increase users' survival, thus emphasizing the importance of evaluation of such hospital-based technologies. This article aims to present a model for the evaluation and incorporation of technology in a teaching hospital that provides high-complexity care. The article describes an approach to methods/processes assessment that can be used easily by any hospital. The model allowed proper health technology assessment (HTA), thereby legitimizing decisions on technology incorporation by the hospital administration with high levels of acceptance and adoption by the clinical staff, suggesting that hospital-based HTA (provided that it is well-structured, with the support of institutional administration) can be a powerful tool for dissemination and valorization of HTA culture in an environment with the widest use and the greatest impact on the health system's budget.
\end{abstract}

Hospitals; Quality of Health Care; Health Technology Evaluation

\author{
Altacílio Aparecido Nunes 1 \\ Luane Marques de Mello 1 \\ Lauro Wichert Ana 1 \\ Paulo Mazzoncini de Azevedo Marques 1 \\ Maria Eulália Lessa Dallora ${ }^{1}$ \\ Edson Zangiacomi Martinez 1 \\ Antonio Pazin Filho 1 \\ Eduardo Barbosa Coelho 1
}

\section{Resumo}

As transições demográfica e epidemiológica tendem a fazer os hospitais cada vez mais necessários à assistência médica. Neles, as tecnologias eficazes, custo-efetivas e seguras, garantem melhor qualidade assistencial e maior sobrevida aos usuários. Assim, a avaliação dessas tecnologias baseada em hospital é prática aconselhável, o que se verifica em todo o mundo. $O$ objetivo deste artigo é apresentar um modelo de avaliação e incorporação de tecnologia em um hospital universitário de alta complexidade. Trata-se de uma descrição de métodos/processos avaliativos que podem ser facilmente adotados por qualquer unidade hospitalar independentemente de sua natureza. Observou-se que o modelo adotado tem possibilitado a adequada avaliação de tecnologias em saúde (ATS), legitimando as decisões de incorporação pelo gestor, com boa aceitação e aderência do corpo clínico. Assim, a ATS em hospital, por empregar processos bem estruturados, consensuais e com apoio da gestão institucional, pode ser uma ferramenta poderosa para disseminação e valorização da cultura de se avaliarem tecnologias em saúde.

Hospitais; Qualidade da Assistência à Saúde; Avaliação de Tecnologias de Saúde 


\section{Introdução}

Os recursos para a saúde são escassos em todo o mundo 1,2. Dados brasileiros demonstram que os gastos no setor representam $8 \%$ do Produto Interno Bruto do país, e que $60 \%$ desse montante são dispendidos em hospitais ${ }^{3,4}$. Tal cenário tende a sofrer incrementos, levando-se em conta os fenômenos das transições demográfica ${ }^{5}$ e epidemiológica 6 , que demandam quase sempre o uso de tecnologias 7 .

A avaliação de tecnologias em saúde (ATS) baseada em hospital não é tema recente. Em 1982, o Comité d'Évaluation et de Diffusion des Innovations Technologiques (Comitê de Avaliação e Disseminação de Inovações Tecnológicas, França) já se preocupava com a avaliação hospitalar de tecnologias e foi o primeiro centro europeu de ATS baseado em hospital 8. Iniciativas dessa natureza progressivamente se espalharam, culminando com a criação de um subgrupo de interesse denominado Hospital Based Health Technology Assessment (Hospital Based HTA) 9 na Health Technology Assessment International (HTAi) 10.

No Brasil, o Ministério da Saúde, objetivando fortalecer e disseminar a ATS dentro dos hospitais e auxiliando gestores na tomada de decisão, criou Núcleos de Avaliação de Tecnologias em Saúde (NATS) em hospitais universitários 11. Para isso, foram apresentados os seguintes argumentos: (a) "A área de ATS é uma novidade no Brasil. Em termos práticos, predomina a escassez de pessoal com formação na área. As instituições de ensino e pesquisa também possuem pouca tradição, tornando-se imprescindivel o investimento na formação de pessoal" 11; (b) as ATS realizadas em nível centralizado não atendem satisfatoriamente as necessidades e particularidades dos níveis de maior uso das tecnologias, que são as organizações de saúde 8,9,10; (c) melhor eficiciência hospitalar reflete em ganhos no nível central, pois o sistema de saúde é composto por grandes complexos assistenciais com peso considerável no impacto orçamentário 8,10,12,13,14.

Considerando tais argumentos, o presente estudo objetiva apresentar um modelo de avaliação e incorporação de tecnologias adotado em um hospital.

\section{Métodos}

Trata-se de uma descrição de processos adotados para avaliação, decisão e incorporação de tecnologias pelo Hospital das Clínicas da Faculdade de Medicina de Ribeirão Preto, Universidade de São Paulo (HCFMRP/USP), que serve como campo de ensino para a Faculdade de Medicina de Ribeirão Preto/USP e outras unidades da universidade. A organização conta com todas as especialidades médicas e com um arsenal tecnológico que propicia assistência e pesquisa de alta qualidade, com impacto na prática clínica.

Desde 2009, o HCFMRP/USP comporta um NATS, no entanto, desde 1995, a instituição já demonstrava interesse pelas tecnologias usadas na saúde e suas repercussões. motivando a criação do Comitê Assessor de Materiais 15, atualmente Comitê de Avaliação de Tecnologias (CAT) 16. O órgão é composto por representantes das áreas críticas no uso de tecnologias e do NATS, com atribuições de receber, analisar/avaliar as solicitações de tecnologias de todos os serviços do hospital.

\section{Resultados}

O CAT se reune quinzenalmente, quando as solicitações são recebidas e formalmente avaliadas. o Comitê as classifica de acordo com um instrumento próprio (Tabela 1), cuja finalidade é ordenar as prioridades.

Para tecnologias classificadas nos níveis 1 e 2, o parecer à superintendência é favorável, não necessitando de análises além das já fornecidas pelo solicitante. Quando classificadas no nível 3, o CAT recorre a análises de produtividade e demanda assistencial do serviço solicitante, além de requerer dados de efetividade e segurança da tecnologia. Caso o solicitante não apresente o exigido, o Comitê solicita ao NATS um breve parecer técnico-científico (PTC-B), a ser apresentado em no máximo trinta dias. Nesse caso, usam-se dados que podem ser fornecidos pelo próprio solicitante, conforme formulário padronizado (Figura 1), ou o parecer é dado de maneira autônoma.

Para tecnologias no nível 4, o CAT exige a realização de estudo mais abrangente, baseando-se no formulário padronizado (Figura 1), preferencialmente revisão sistemática com metanálise demonstrando eficácia e segurança, além de estudos econômicos, geralmente realizados conjuntamente entre o NATS e especialistas do serviço. Caso haja alguma pesquisa em andamento sobre a tecnologia solicitada, os resultados são aguardados e posteriormente avaliados em todos os seus aspectos. O caminho percorrido por qualquer solicitação de tecnologia dentro do HCFMRP/USP segue os passos mostrados na Figura 2.

Em caso de solicitação ao CAT de avaliação da tecnologia classificada no nível 4 de prioridade, o processo pode ser observado na Figura 3. Nesse 
Classificação de prioridades de atendimento às solicitações de tecnologias em saúde recebidas pelo Comitê de Avaliação de Tecnologias, natureza das tecnologias e respectivos percentuais. Hospital das Clínicas, Faculdade de Medicina de Ribeirão Preto, Universidade de São Paulo, São Paulo, Brasil.

\begin{tabular}{|c|c|c|c|}
\hline Nível de prioridade & Critério & Tecnologia & Percentuais \\
\hline 1 & $\begin{array}{c}\text { Urgente, risco imediato de } \\
\text { interrupção do serviço/atividade } \\
\text { assistencial }\end{array}$ & $\begin{array}{l}\text { Equipamentos e dispositivos médicos } \\
\text { de diagnóstico e suporte à vida }\end{array}$ & 10 \\
\hline 2 & $\begin{array}{c}\text { Substituição de tecnologia já } \\
\text { existente em razão de inviabilidade } \\
\text { de recuperação ou por obsolescência. } \\
\text { Visa à manutenção de procedimentos } \\
\text { e/ou atividades assistenciais já } \\
\text { implantadas }\end{array}$ & $\begin{array}{c}\text { Equipamentos e dispositivos } \\
\text { médicos de diagnóstico (imagem) e } \\
\text { monitoramento }\end{array}$ & 20 \\
\hline 3 & $\begin{array}{c}\text { Tecnologia adicional às já existentes } \\
\text { no setor/serviço. Visa a cobrir } \\
\text { demanda existente (mas reprimida) } \\
\text { - expansão de oferta ou melhoria da } \\
\text { qualidade terapêutica/diagnóstica }\end{array}$ & $\begin{array}{l}\text { Equipamentos médicos de } \\
\text { diagnósticos e terapêuticos, } \\
\text { medicamentos, kits diagnósticos }\end{array}$ & 40 \\
\hline 4 & $\begin{array}{l}\text { Incorporação de nova tecnologia em } \\
\text { substituição a tecnologia obsoleta } \\
\text { ou para implantação e oferta de } \\
\text { novos procedimentos e/ou atividades } \\
\text { assistenciais }\end{array}$ & $\begin{array}{l}\text { Equipamentos e dispositivos médicos } \\
\text { diagnósticos e terapêuticos de alta } \\
\text { densidade tecnológica e alto custo } \\
\text { financeiro }\end{array}$ & 30 \\
\hline
\end{tabular}

caso há importante participação da Unidade de Pesquisa Clínica e do serviço de engenharia clínica. Essas associações no processo têm garantido que pelo menos duas novas tecnologias sejam anualmente incorporadas.

\section{Discussão}

As ATS podem ser consideradas sob três pontos de vista 10,17,18,19, como se esclarece a seguir.

O nível micro tem como centro o usuário de serviço de saúde, ancorado em aspectos particularizados da condição ou doença apresentada pelo paciente, não sendo considerado o "coletivo" em nenhum aspecto; por isso, tal modelo não deve ser adotado, pois geralmente não há dados suficientes que justifiquem o uso da tecnologia. Outro ponto negativo é o da indução da judicialização, fenômeno que tem ganhado força em nosso meio e que reforça ainda mais o sentido de injustiça, ao beneficiar um único paciente em detrimento do coletivo.

O nível superior (macro), no qual as avaliações se prestam a subsidiar os gestores centrais na tomada de decisão, ou seja, o foco é o sistema de saúde como um todo. Esse tem sido o modelo que compreensivelmente mais opera no Brasil, até mesmo pela preocupação e introdução recente dessa cultura em nosso país. No entanto, tem-se observado uma mudança desse paradigma com o estímulo e indução de criação de núcleos de ATS localizados em hospitais, evidenciando outro modelo, o de nível intermediário ou "meso".

No nível meso, as avaliações subsidiam decisões a serem tomadas nas organizações. Esse modelo tem muitas vantagens e parece resultar em maior rapidez sem perda da qualidade e confiabilidade, fato que é concordante com outros que têm usado modelos semelhantes 8,13,20.

\section{Conclusões}

Apresentou-se um modelo de ATS baseado em hospital que tem gerado bons resultados. A metodologia empregada no HCFMRP/USP assemelha-se com o que se propõe no modelo de mini-HTA 21, com a vantagem de ser mais simples operacionalmente e contar com interações importantes. Os passos processuais adotados na instituição são simples, acessíveis e de conhecimento de todos os serviços e departamentos; 
Figura 1

Formulário de auxílio à realização de parecer técnico-científico (PTC-B) pelo Núcleo de Avaliação de Tecnologias em Saúde, Hospital das Clínicas, Faculdade de Medicina de Ribeirão Preto, Universidade de São Paulo, São Paulo, Brasil.

N.A.T.S. - Núcleo de Avaliação de Tecnologias em Saúde

Solicitação de Parecer Técnico-Científico I

1. Solicitante: _ Consultor (especialista) do PTC:

2. Descrição e justificativa da tecnologia (material/equipamento/procedimento):

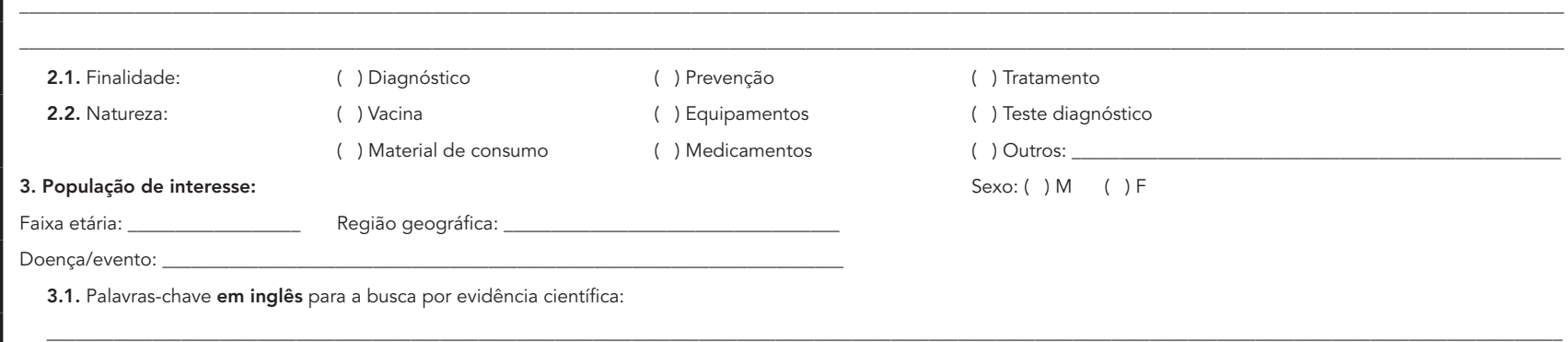

\section{Descreva como a tecnologia será utilizada:}

4.1. Palavras-chave em inglês que descrevem a tecnologia a ser utilizada:

5. Quais os tipos de estudos primários que permitem avaliar a tecnologia em questão?
( ) Caso-controle
( ) Estudo de teste diagnóstico
( ) Estudo clínico controlado randomizado

6. Tecnologias alternativas e o padrão ouro para comparação:

6.1. Palavras-chave em inglês que descrevem o padrão ouro de comparação:

7. Qual(is) o(s) resultado(s)/desfecho(s) esperado(s) com a tecnologia solicitada?

7.1. Palavras-chave em inglês que descrevem o resultado/desfecho:

Data: _ I -

Assinatura do solicitante

Para uso do N.A.T.S.:

Registro na ANVISA/MS （） Sim （） Não

Indicação de fontes
( ) PubMed/MEDLINE
( ) Scopus
( ) SILABUS
( ) LILACS/BIREME
( ) Pdq
( ) BIOSIS
( ) Embase
( ) HealthStar
( ) WoS - Web of Science
( ) Hta/Nihr
( ) Life
( ) HAPI
( ) Revisões sistemáticas da Cochrane
( ) Clin
( ) $\mathrm{PAHO}$
( ) PsicLit
( ) BEHA
( ) SCiELO
( ) CancerLit
( ) PEDro
( ) Outro(s):

Data: _ _ _ 1 
Figura 2

Fluxograma simplificado do processo de solicitação, análise e avaliação de tecnologias em saúde no Comitê de Avaliação de Tecnologias (CAT), Hospital das Clínicas, Faculdade de Medicina de Ribeirão Preto, Universidade de São Paulo, São Paulo,

Brasil.

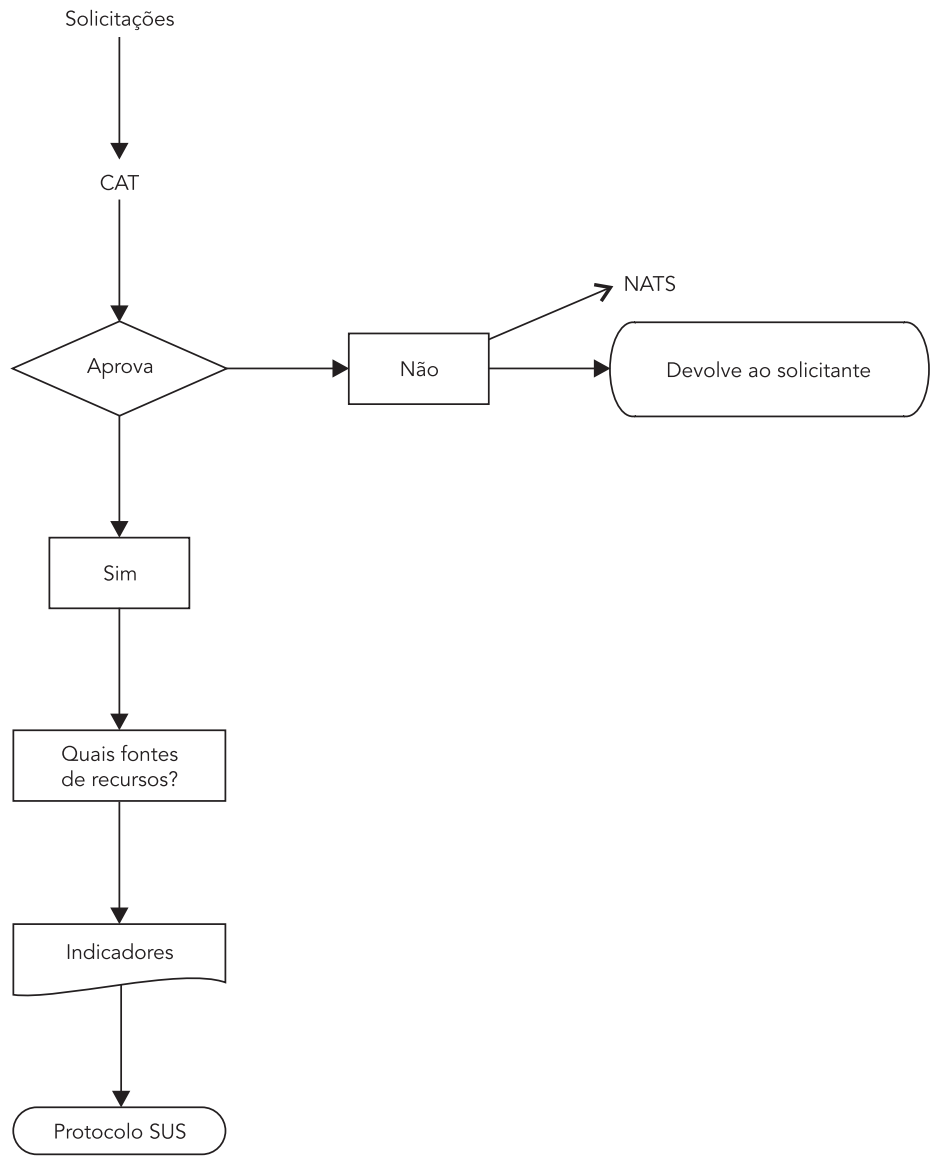

NATS: Núcleos de Avaliação de Tecnologias em Saúde; SUS: Sistema Único de Saúde.

por isso, são bem aceitos, ao contrário do modelo avaliado com a utilização do mini-HTA 22. A ATS baseada em hospital não substitui, e nem se pretende que substitua, as avaliações completas, no entanto deve ser valorizada não só por suas particularidades, mas, sobretudo, por ser no cenário hospitalar, onde reside o uso das tecnologias de maior impacto financeiro e de expectativa de desfechos positivos. Segundo McGregor 13, a implementação de unidade de ATS - in house pode ser útil para criar um contexto organizacional positivo, facilitando o uso de evidência científica, a fim de sustentar a prática clínica e a decisão gerencial em hospitais. 
Figura 3

Fluxograma do processo de avaliações de tecnologias (nível de prioridade 4) em saúde realizadas pelo Núcleo de Avaliação de Tecnologias em Saúde (NATS), demandadas pelo Comitê de Avaliação de Tecnologias, Hospital das Clínicas, Faculdade de Medicina de Ribeirão Preto (HCFMRP), Universidade de São Paulo, São Paulo, Brasil.

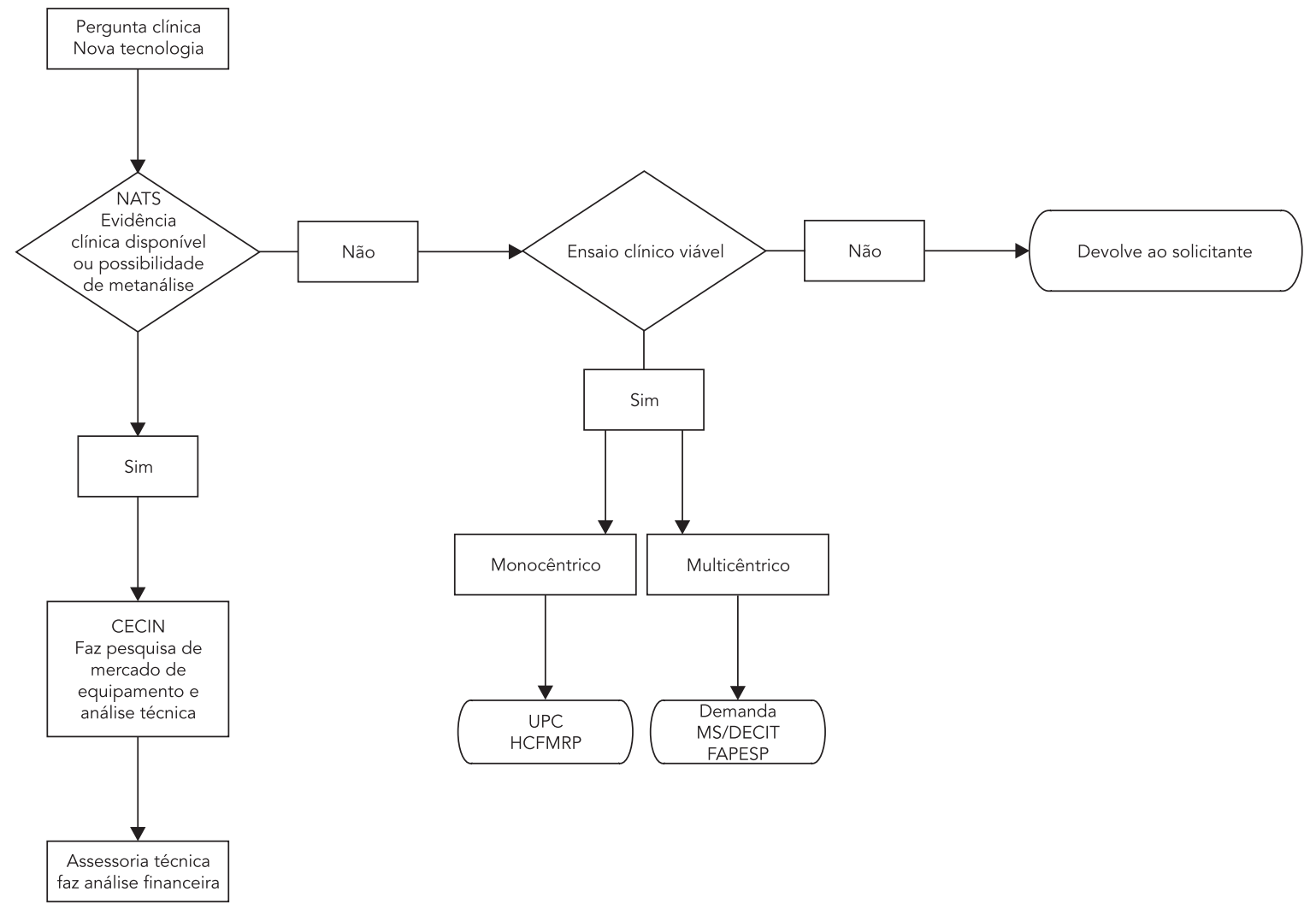

CECIN: Centro de Engenharia Clínica; DECIT: Departamento de Ciência e Tecnologia; FAPESP: Fundação de Amparo à Pesquisa do Estado de São Paulo; MS: Ministério da Saúde; UPC: Unidade de Pesquisa Clínica. 


\section{Resumen}

Las transiciones demográficas y epidemiológicas tienden a convertir a los hospitales en servicios cada vez más necesarios. En ellos las tecnologías eficaces, rentables y seguras, garantizan una mejor calidad de atención y una supervivencia más amplia a los usuarios, por lo que se aconseja evaluar, basándose en la práctica hospitalaria, los recursos existentes alrededor del mundo. El propósito de este artículo es presentar un modelo de evaluación e incorporación de tecnología en un hospital universitario de alta complejidad. Esta es una descripción de métodos/procesos de evaluación que pueden ser utilizados por cualquier unidad hospitalaria independientemente de su naturaleza. Se observó que el modelo adoptado ha permitido la evaluación de la tecnología de salud, ha legitimado las decisiones de su incorporación por parte del administrador, con una buena aceptación y adhesión del personal clínico, indicando que la evaluación de tecnologías en salud (ETS) en un hospital, empleando procesos bien estructurados, de consenso y con el apoyo de la gestión institucional, puede ser una poderosa herramienta para la difusión y valorización de la cultura en ETS.

Hospitales; Calidad de Atención de Salud; Evaluación de Tecnologías de Salud

\section{Colaboradores}

A. A. Nunes contribuiu no levantamento de dados, revisão bibliográfica, redação, revisão e submissão do manuscrito. L. M. Mello, L. W. Ana, P. M. A. Marques, E. Z. Martinez, A. Pazin Filho e E. B. Coelho colaboraram na redação e revisão do manuscrito. M. E. L. Dallora participou no levantamento de dados documentais, redação e revisão do manuscrito.

\section{Agradecimentos}

À FAEPA-HCFMRP/USP pelo apoio logístico.

\section{Conflito de interesses}

Não declarado.

\section{Referências}

1. Yang BM. The future of health technology assessment in healthcare decision making in Asia. Pharmacoeconomics 2009; 27:891-901.

2. Tan-Torres T. Technology assessment in developing countries. World Health Forum 1995; 16:74-6.

3. Ribeiro JM. Desenvolvimento do SUS e racionamento de serviços hospitalares. Ciênc Saúde Coletiva 2009; 14:771-82.

4. Planejamento e Organização de Instituições de Saúde. Apuração dos custos de procedimentos hospitalares: alta e média complexidade. Projeto Reforsus. [CD-ROM]. São Paulo: Planejamento e Organização de Instituições de Saúde; 2002.

5. Mendes ACG, Sá DA, Miranda GM, Lyra TM, Tavares RA. The public healthcare system in the context of Brazil's demographic transition: current and future demands. Cad Saúde Pública 2012; 28:955-64.

6. Bittar OJNV, Mendes JDV, Magalhães A. Administração de sistemas e serviçõs de saúde: competências exigidas na atualidade. Revista de Administração Hospitalar e Inovação em Saúde 2011; 7:30-44.
7. Carolina MS, Gustavo LF. Epidemiological transition: model or illusion? A look at the problem of health in Mexico. Soc Sci Med 2003; 57:539-50.

8. Baffert S, Charpentier E, Fay AF. HTA from the point of view of the hospital: specificities and examples (CEDIT). Ital J Pub Health 2005; 2 Suppl $1: 27$.

9. Health Tecnology Assessment International. HTAi interest sub-group on Hospital Based HTA. http:// www.htai.org/index.php?id=565 (acessado em 20/ dez/2012).

10. Cicchetti A, Marchetti M, Dibidino R, Corio M. Hospital based health technology assessment. Worldwide survey. HTAi interest sub-group Hospital Based HTA 2008. http://www.htai.org/fileadmin/ HTAi_Files/ISG/HospitalBasedHTA/2008Files/ HospitalBasedHTAISGSurveyReport.pdf (acessado em 20/Dez/2012). 
11. Departamento de Ciência e Tecnologia, Secretaria de Ciência, Tecnologia e Insumos Estratégicos, Ministério da Saúde. Chamada pública para implantação de núcleos de Avaliação de Tecnologias em Saúde em hospitais de ensino - 2009. http:// portal.saude.gov.br/portal/arquivos/pdf/edital_ nucleos_2009.pdf (acessado em 20/Dez/2012).

12. Dopson S, Fitzgerald L. Knowledge to action? Evidence based health care in context. Oxford: Oxford University Press; 2005.

13. McGregor M, Brobhy JM. End-user involvement in health technology assessment (HTA) development: a way to increase impact. Int J Technol Assess Health Care 2005; 21:263-7.

14. Rosen R, Gabbey J. Linking health technology assessment to practice. BMJ 1999; 319:1-3.

15. Secretaria de Estado da Saúde de São Paulo. Portaria HCRP-73, de 19 de abril de 1995. Diário Oficial do Estado 1995; 1 jun.

16. Secretaria de Estado da Saúde de São Paulo. Portaria HCRP-111, de 25 de maio 2011. Diário Oficial do Estado 2011; 26 mai.

17. Gutiérrez-Ibarluzea I. Personalised health care, the need for reassessment. A HTA perspective far beyond cost-effectiveness. Ital J Public Health 2012; 9(4). http://ijphjournal.it/article/view/8653.
18. Postma MJ, Boersma C, Vandijck D, Vegter S, Le $\mathrm{HH}$, Annemans L. Health technology assessments in personalized medicine:illustrations for costeffectiveness analysis. Expert Rev Pharmacoecon Outcomes Res 2011; 11:367-9.

19. Catananti C, Cicchetti A, Marchetti M. Hospitalbased Health Technology Assessment: the experience of Agostino Gemelli University Hospital's HTA Unit. Ital J Public Health 2005; 2(2). http:// ijphjournal.it/article/view/5980.

20. Purmonen TT, Auvinen PK, Martikainen JA. Budget impact analysis of trastuzumab in early breast cancer: a hospital district perspective. Int J Technol Assess Health Care 2010; 26:163-9.

21. Ehiers L, Vestergaard M, Kidholm K, Bonnevie $\mathrm{B}$, Pedersen PH, JØrgensen $\mathrm{T}$, et al. Doing minihealth technology assessments in hospitals: a new concept of decision support in health care? Int J Technol Assess Health Care 2006; 22:295-301.

22. Kidholm K, Ehlers L, Korsbek L, Kjaerby R, Beck M. Assessment of the quality of mini-HTA. Int J Technol Assess Health Care 2009; 25:42-8.

Recebido em 13/Jan/2013

Versão final reapresentado em 31/Mai/2013

Aprovado em 04/Jul/2013 